\title{
Application of Algebraic Combinatorics to Finite Spin Systems
}

\author{
W. FLOREK ${ }^{*}$ \\ Institute of Physics, A. Mickiewicz University \\ Umultowska 85, 61-614 Poznań, Poland
}

(Received June 11, 2001; in final form July 13, 2001)

\begin{abstract}
A finite spin system invariant under a symmetry group $G$ is a very illustrative example of a finite group action on mappings $f: X \rightarrow Y(X$ is a set of spin carriers, $Y$ contains spin projections for a given spin number $s)$. Orbits and stabilizers are used as additional indices of the symmetry adapted basis. Their mathematical nature does not decrease a dimension of a given eigenproblem, but they label states in a systematic way. It allows construction of general formulas for vectors of symmetry adapted basis and matrix elements of operators commuting with the action of $G$ in the space of states. The special role is played by double cosets, since they label nonequivalent (from the symmetry point of view) matrix elements $\langle x|\mathcal{H}| y\rangle$ for an operator $\mathcal{H}$ between Ising configurations $|x\rangle,|y\rangle$. Considerations presented in this paper should be followed by a detailed discussion of different symmetry groups (e.g.) cyclic or dihedral ones) and optimal implementation of algorithms. The paradigmatic example, i.e. a finite spin system, can be useful in investigations of magnetic macromolecules like $\mathrm{Fe}_{6}$ or $\mathrm{Mn}_{12}$ acetate.
\end{abstract}

PACS numbers: 02.20.Bb, 75.10.Jm, 75.75.+a

\section{Introduction}

Finite spin models have been used in condensed matter physics for many years $[1,2]$ and have been applied to various models of magnetic materials: from the one-dimensional ferromagnetic Ising model to the three-dimensional Heisenberg antiferromagnets. Different extrapolation techniques have been introduced and many different parameters have been calculated: ground state energy, specific heat, spin correlations in excited states, etc. [3]. Recent developments and

*e-mail: Wojciech.Florek@spin.amu.edu.pl 
discoveries have stimulated interests in finite spin models, since they can be applied to meso- and nanoscopic systems, especially magnetic macromolecules with well-determined symmetry groups like $\left[\mathrm{NaFe}_{6}\left(\mathrm{OCH}_{3}\right)_{12}\left(\mathrm{C}_{17} \mathrm{H}_{15} \mathrm{O}_{4}\right)_{6}\right] \mathrm{ClO}_{4}\left(\mathrm{Fe}_{6}\right)$, $\left[\mathrm{Fe}_{10}\left(\mathrm{OCH}_{3}\right)_{20}\left(\mathrm{C}_{2} \mathrm{H}_{2} \mathrm{O}_{2} \mathrm{Cl}\right)_{10}\right]\left(\mathrm{Fe}_{10}\right)$ or $\mathrm{Mn}_{12}$ acetate $[4,5]$. In modeling of infinite magnets much more efforts have been devoted to small spin numbers (the Ising models or $s=1 / 2,1$ ), but with a very large number of nodes (up to millions in the Monte Carlo methods, see for example [6, 7]). On the contrary, macromolecules contain several (up to $N=30$ ) magnetic ions with relatively large spin numbers (up to $s=5 / 2$ ), which also leads to a very large number of states even for a small number of magnetic ions $N$. Moreover, this number increases rapidly with increasing $N$ and $s$. However, group-theoretical and combinatorial methods yield decomposition of a space of states into subspaces with relatively small dimensions and for small, and rather simple symmetry groups like cyclic or dihedral ones, it can be quite easy. Therefore, magnetic properties of macromolecules can be investigated very deeply and model parameters can be fitted in a more accurate way. This paper, based on the results of some earlier papers [8-10], has been meant as one of the steps necessary in the study considerations of small (nanoscopic) rings with a relatively large spin number. However, the combinatorial methods and structures are also applied to investigation of magnetic properties in the thermodynamic limit [11].

It should be also stressed that the approach described and solutions suggested can be used in any problem where a Hamiltonian symmetry group $G$ (or, at least, its subgroup) permutes basis vectors. It means that a permutation representation $P$ of $G$ is determined or, in other words, a group action of $G$ on a basis $\mathcal{B}$ of quantum space of states is introduced. This immediately gives rise to such structures and concepts as transitive representations, orbits, stabilizers, double cosets, etc. Permutation representations themselves constitute a special case of induced representation and are applied in many branches of physics [12]. Some problems related to permutation and transitive representations were studied by Lulek et al. $[10,13,14]$ and their results, in a various extent, are used here.

The aim of this paper is to present and discuss a combinatorial structure of finite spin models. Such investigations lead to general analytical formulas for the irreducible basis and matrix elements of any operator commuting with all symmetry operators $P(g), g \in G$. The most important is a Hamiltonian of the system in question, but also other operators can be taken into account. For example, in the case of isotropic Heisenberg magnets the squared total spin operator $S^{2}$ is considered. Since $\boldsymbol{S}^{2}$ commutes with the isotropic Heisenberg Hamiltonian, then the total spin $S$ can be used as an additional label of states. The considerations presented here should be followed by careful implementation of equations and methods in numerical procedures $[15,16]$.

The methods applied originate from algebraic combinatorics and are closely related to group theory. Since some structures, like double cosets, have been rather 
rarely used in physics, so we start with their presentation (Secs. 2 and 3). Next the formulas obtained earlier [9] are discussed and modified applying the notion of double cosets. The special cases are also discussed. Some modifications related to the total magnetization and the notion of partitions are considered in Sec. 5. The structures introduced and formulas obtained are applied to a very simple system of four spins $s=1$ with the symmetry group $G=D_{4}$ (Sec. 6). All results obtained for the dihedral groups $D_{N}$ are presented in more detail in the second part of this work [17].

\section{Group action}

This paper is based on some methods of algebraic combinatorics [18] and the theory of groups and their representations $[19,20]$. We would like to present a brief report on the main objects and common objects of these two theories, because of confusing notions used in different handbooks.

\subsection{Finite group action on sets}

Let $G$ be a finite group and let $X$ be a finite set. A group of all bijections $\sigma: X \rightarrow X$ is called the symmetric group on $X$ and denoted $\Sigma_{X}$. Sets with the same cardinality $N=|X|$, where $|X|$ denotes the number of elements of the finite set $X$, have symmetric groups isomorphic with the symmetric group $\Sigma_{N}$ consisting of all permutations $\sigma$ of $N$-element set $\underline{N}=\{1,2, \ldots, N\}$.

An action of $G$ on a (finite) set $X$ is denoted ${ }_{G} X$ and can be introduced in two equivalent ways. The first is based on the notion of an external action (the multiplication rule): there is such a mapping $G \times X \rightarrow X$, that to each pair ( $g, x)$ an element $g x \in X$ is assigned and

$$
\forall g_{1}, g_{2} \in G, x \in X: \quad g_{1}\left(g_{2} x\right)=\left(g_{1} g_{2}\right) x \quad \text { and } \quad e_{G} x=x,
$$

where $e_{G}$ denotes the unit element of $G$. The second definition introduces the notion of the permutation representation $P$ of $G$ as a homomorphism

$$
P: G \rightarrow \Sigma_{X}: g \mapsto P(g),
$$

so $P\left(g_{1} g_{2}\right)=P\left(g_{1}\right) P\left(g_{2}\right)$. Identifying $g x$ with $P(g) x$ one obtains the equivalence of these definitions. Since $P$ is not, in general, a monomorphism then its kernel Ker $P$ may be non-trivial and its image $\operatorname{Im} P$, denoted also by $\bar{G}$, is isomorphic with $G /$ Ker $P$. Sometimes this image is considered instead of a full symmetry group $G$ : for example in the case of spin rings we omit the horizontal symmetry plane and consider the dihedral group $D_{N}$ instead of the group $D_{N h}$. This group is important, for example, in constructing symmetry coordinates of vibrating molecules or crystals, when movements along the $z$ axis have to be classified, too.

The action ${ }_{G} X$ gives rise to two important structures: an orbit $G(x)=$ $\{x \mid g \in X\}$, containing all elements $y \in X$ which can be obtained from $x$ and a 
stabilizer $G_{x}=\{g \mid g x=x\}$, consisting of group elements leaving $x$ invariant. It is easy to show that [18]

$$
|G(x)|=|G| /\left|G_{x}\right| \text {. }
$$

Stabilizers of elements from the same orbit are conjugated, since for $y=g x$ and $h \in G_{x}$ one obtains $\left(g h g^{-1}\right) y=(g h) x=y$. This property is the crucial point of similarity [18] between the action of $G$ on an orbit $G(x)$ and the natural action of $G$ on left cosets $G / G_{x}$

$$
G \times G / G_{x} \rightarrow G / G_{x}:\left(g^{\prime}, g G_{x}\right) \mapsto\left(g^{\prime} g\right) G_{x} .
$$

Since orbits are equivalence classes then $X$ is decomposed into a disjoint sum of orbits

$$
X=\bigcup^{t \in \mathcal{T}} G(t)
$$

where the transversal $\mathcal{T}$ contains (arbitrarily chosen) representatives of orbits. If $X=G(x)$ then we say that the action is transitive and $G$ acts transitively on $X$. Due to the above dissection of $X$ into orbits each transitive action ${ }_{G} G(x) \approx_{G}\left(G / G_{x}\right)$ can be considered separately. It corresponds to a decomposition of the permutation representation $P$ into a direct sum of transitive representations $P \downarrow G(t), t \in \mathcal{T}$ (subactions ${ }_{G} G(t)$ ). Since orbits are characterized by their stabilizers $G_{t}$, then transitive representations are as usual labelled by $G_{t}$ and denoted $R^{G: G_{t}}[13]$, so $P=\bigoplus_{t \in \mathcal{T}} R^{G: G_{t}}$. Orbits $G(x)$ and $G(y)$ with conjugated stabilizers, i.e. when there exists such $g \in G$ that $G(y)=g G(x) g^{-1}$, are in the same type (they form the so-called stratum), since both actions, $G G(x)$ and ${ }_{G} G(y)$, are similar to the action ${ }_{G}(G / U)$, where $U \subset G$ represents a class $\widetilde{U}$ of conjugated subgroups of $G$. Let $\mathcal{U}$ denotes a transversal (a set of representatives) of classes of conjugated subgroups. Then each permutation representation $P$ can be decomposed into a direct sum of transitive actions

$$
P=\bigoplus_{U \in \mathcal{U}} n\left(R^{G: U}, P\right) R^{G: U}
$$

where the multiplicity $n\left(R^{G: U}, P\right)$ equals a number of orbits in the type $\widetilde{U}$ or, in other words, a length of the $\tilde{U}$-stratum.

The group $G$ is supposed to be finite [18] and hence the set of the conjugacy classes of subgroups of $G$ is finite and partially ordered with respect to the inclusion order

$$
\widetilde{U} \subset \widetilde{V} \Leftrightarrow \exists U \in \widetilde{U}, V \in \widetilde{V}: U \subset V .
$$

This set is in fact a lattice, since there is the maximal element, $\widetilde{G}=\{G\}$, and the minimal one - the class $\widetilde{E}$ containing the trivial subgroup $E=\left\{e_{G}\right\}$. The first determines one-element orbit $G(x)=\{x\}$ with $g x=x$ for all $g \in G$, whereas the latter defines the (left) regular action and the regular orbit consisting of $|G|$ elements with the trivial stabilizer: $G_{y}=\left\{e_{G}\right\}$ for all $y \in G(x)$. Regular orbits form the so-called generic stratum. 
All transitive actions ${ }_{G}(G / U)$ (representations $\left.R^{G: U}\right), U \in \widetilde{U}$, can be completely investigated for a finite group $G$. In particular cases, when the multiplication rule in $G$ can be given analytically, some general formulas can be derived. To apply the obtained results to a given action ${ }_{G} X$ we have to fix representatives $U$ of conjugacy classes $\widetilde{U}$ and representatives $g_{j}$ of left cosets $g_{j} U(1 \leq j \leq|G / U|$, $\left.g_{1}=e_{G}\right)$. For a given action ${ }_{G} X$ all orbits can be generated/constructed in such a way that their transversal $\mathcal{T}$ consists of elements $t$ with stabilizers $G_{t}=U$ (it means that $t \in \mathcal{T} \Rightarrow G_{t} \in \mathcal{U}$ ) and orbit elements are uniquely identified by coset representatives $g_{j}$, i.e. labelled by the index $j$.

\subsection{Problem linearization}

In many physical applications a set $X$ is, or at least can be formally treated as, a base $\mathcal{B}$ of an $|X|$-dimensional unitary space $L$. The operators $P(g)$ permute basis vectors $\mathcal{B} \ni|x\rangle \equiv x \in X$, so they form a unitary (vector) representation of $G$. The decomposition of $X$ into orbits $G(x)$ corresponds to the decomposition of $\mathcal{B}$ into disjoint subbases $\mathcal{B}_{t}, t \in \mathcal{T}$, and, therefore, to the decomposition of $L$ into a direct sum of subspaces $L_{t}$. Since these subspaces are invariant under the action of operators $P(g)$, then $P$ is decomposed into direct sum of transitive representations $R^{G: G_{t}}$ or, more precisely, Eq. (2) is also valid if $P$ and $R^{G: U}$ are treated as vector representations.

Transitive representations, which are "building blocks" of permutation representations, are, in a general case, reducible and, therefore, can be decomposed into irreducible representations (irreps) $\Gamma$ of $G$

$$
R^{G: U}=\bigoplus_{\Gamma} n\left(\Gamma, R^{G: U}\right) \Gamma
$$

In the special cases one obtains

$$
R^{G: G}=\Gamma_{0} \quad \text { and } \quad R^{G: E}=\bigoplus_{\Gamma}[\Gamma] \Gamma,
$$

where $\Gamma_{0}$ denotes the unit representation and $[\Gamma]$ is the dimension of an irrep $\Gamma$. In intermediate cases the multiplicity $n\left(\Gamma, R^{G: U}\right)$ takes values from 0 through $[\Gamma]$. The actual formula is presented in Sec. 2.4.

\subsection{Action on mappings}

Let $X$ and $Y$ be finite sets and let $Y^{X}$ be a set of all mappings from $X$ into $Y$

$$
Y^{X}=\{f \mid f: X-Y\} .
$$

An action ${ }_{G} X$ can be raised to $Y^{X}$ in the following way:

$$
G \times Y^{X} \rightarrow Y^{X}:(g, f) \mapsto f^{\prime}=f \circ P(g)^{-1},
$$

i.e. $(g, f)$ is mapped onto $f^{\prime}$ determined as $f^{\prime}(x)=f\left(g^{-1} x\right)$. 
All structures and properties presented in the previous sections can be immediately applied to this action. In addition, we can exclude certain types of orbits, which means that not all subgroups $U$ can be stabilizers of mappings. Any subgroup $U \subset G$ decomposes $X$ into disjoint orbits $X_{j} \subset X$. This decomposition defines a Young subgroup $\prod_{j} \Sigma_{X_{j}}$ of the symmetric group $\Sigma_{X}$. The subgroup $U$ can be a stabilizer of a mapping $f \in Y^{X}$ if and only if [18]

$$
\bar{U}=\bar{G} \cap \prod_{j} \Sigma_{X_{j}}
$$

It is easy to check that both trivial subgroups $G$ and $E$ can be stabilizers of mappings. In the case of $G=D_{N}$ acting on an $N$-element ring this theorem excludes, among others, the cyclic subgroup $U=C_{N}$. Nodes of the ring form a regular orbit of $C_{N}$, so the Young subgroup is identical with $\Sigma_{X}$ and, therefore, $\bar{D}_{N} \cap \Sigma_{X}=\bar{D}_{N} \neq \bar{C}_{N}$

There are also other possible actions on $Y^{X}$ but this one is important in the case of finite spin systems. The roles of $X$ and $Y$ are played by the set $\underline{N}$ of $N$ nodes (sites, spin carriers) and the set $[-s, s]=\{m \mid-s \leq m \leq s\}$ of spin projections for a given spin number $s$, respectively. Mappings $\mu: \underline{N} \rightarrow[-s, s]$ are in a one-to-one correspondence with the so-called Ising configurations

$$
\mu=\left|m_{1} m_{2} \ldots m_{N}\right\rangle, \quad m_{j}=\mu(j), \quad 1 \leq j \leq N .
$$

These states form a natural basis $\mathcal{B}$ of the quantum space $L$ of states with $\operatorname{dim} L=(2 s+1)^{N}$. In a general case, however, these states are not eigenvectors of the Heisenberg-like Hamiltonians. Therefore, it is necessary to solve an eigenproblem with thousands or millions equations which, even using contemporary computers, is almost impossible. This is especially important when one is interested not only in eigenvalues (e.g. the ground state energy), but also wants to investigate eigenvectors to determine, for example, spin correlations. The problem is partially solved by taking into account operators commuting with a given Hamiltonian: good quantum numbers as the total magnetization $S^{z}$ and irreps of the Hamiltonian symmetry group $G$. Application of irreps leads to the concept of symmetry adapted (or irreducible) basis

$$
|\Gamma r \gamma\rangle=\sum_{\mu} b_{\mu}^{\Gamma r \gamma} \mu
$$

where $\Gamma$ is an irrep of $G, \gamma=1,2, \ldots,[\Gamma]$ labels its vectors, and the so-called repetition index $r=1,2, \ldots, n(\Gamma)$ distinguishes copies of $\Gamma(n(\Gamma)$ denotes a number of these copies). Due to the Schur Lemma [19] eigenvectors of an operator commuting with symmetry operators are linear combinations of these vectors with $\Gamma$ and $\gamma$ fixed, so they can be written as $\sum_{r=1}^{n(\Gamma)} \alpha_{r}|\Gamma r \gamma\rangle$ and, therefore, one obtains eigenproblems with dimensions $n(\Gamma)$. They still can be very large and much efforts have been devoted to replace a single index $r$ by a series of indices with a well-defined physical or/and mathematical meaning $[10,12,13,21$, and the references quoted 
in these papers]. The first type is constituted by, mentioned earlier, good quantum numbers, especially those corresponding to operators with the same (or higher) symmetry as the Hamiltonian and with the known eigenvalues (like the total spin $S$ or the magnetization $M$ ). As a rule, the others do not lead to smaller eigenproblems but their mathematical nature enables us to write general analytical formulas for matrix elements. This paper is mainly devoted to such indices. The operator eigenproblem is the standard numerical task, so it is not discussed here.

\subsection{Reduction coefficients}

An irrep $\Gamma$ of a group $G$ is, as a rule, a reducible representation of its subgroup $U \subset G$. Hence, $\Gamma$ can be decomposed into a direct sum

$$
\Gamma \downarrow U=\bigoplus_{\Xi} n(\Xi, \Gamma \downarrow U) \Xi
$$

over irreps $\Xi$ of a subgroup $U$. In the same way the carrier space $L_{\Gamma}$ of $\Gamma$ decomposes into a direct sum of subspaces $L_{\Xi r}, r=1,2, \ldots, n(\Xi, \Gamma \downarrow U)$. The basis related to this decomposition can be written as

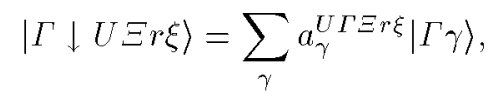

where $\gamma$ and $\xi$ label basis vectors of $L_{\Gamma}$ and $L_{\Xi}$, respectively. The numbers $a_{\gamma}^{U \Gamma \Xi r \xi}$ are referred to as the reduction coefficients.

In the problem discussed in this paper we are interested in the reduction of irreps appearing in decompositions of transitive representations $R^{G: U}$. Due to the Frobenius reciprocity theorem $[13,18]$

$$
n\left(\Gamma, R^{G: U}\right)=n\left(\Xi_{0}, \Gamma \downarrow U\right) .
$$

Moreover, $0 \leq n\left(\Gamma, R^{G: U}\right) \leq[\Gamma]$. Therefore, we are interested in the reduction coefficients for a given $U$ and $\Xi=\Xi_{0}$ ( $\xi$ may be omitted $)$

$$
A_{\gamma}^{U \Gamma r}=a_{\gamma}^{U \Gamma \Xi_{0} r}
$$

with $r$ no larger than $[\Gamma]$. These numbers appear in the formula (7) written for $\Xi=\Xi_{0}$

$$
\left|\Gamma \downarrow U \Xi_{0}\right\rangle=\sum_{\gamma} A_{\gamma}^{U \Gamma r}|\Gamma \gamma\rangle
$$

Since $P(u)\left|\Gamma \downarrow U \Xi_{0}\right\rangle=\left|\Gamma \downarrow U \Xi_{0}\right\rangle$ for $u \in U$ then the same equality is satisfied by the r.h.s. of Eq. (9). Taking into account that

$$
P(u) \sum_{\gamma} A_{\gamma}^{U \Gamma r}|\Gamma \gamma\rangle=\sum_{\gamma, \gamma^{\prime}} A_{\gamma}^{U \Gamma r} \Gamma_{\gamma^{\prime} \gamma}(u)\left|\Gamma \gamma^{\prime}\right\rangle
$$

one obtains (for $u \in U$ )

$$
A_{\gamma}^{U \Gamma r}=\sum_{\gamma^{\prime}} \Gamma_{\gamma \gamma^{\prime}}(u) A_{\gamma^{\prime}}^{U \Gamma r}
$$


It has to be stressed that $\Gamma_{0} \downarrow U=\Xi_{0}$ for all $U \subset G$, so $\Gamma_{0}$ appears exactly once in the decomposition of each $R^{G: U}$ and $A^{U \Gamma_{0}}=1$ (the indices $\gamma$ and $r$ can be omitted).

\section{Double cosets}

For a given group $G$ and its two subgroups $V$ and $U$ a double coset determined (represented) by an element $g \in G$ is a set $[18,19]$

$$
V g U=\left\{v g u^{-1} \mid v \in V, u \in U\right\}
$$

A set of all double cosets is denoted $V \backslash G / U$. If $V$ (or $U$ ) is a trivial subgroup $E=\left\{e_{G}\right\}$ then a left (respectively, right) coset is obtained, so double cosets constitute a simple, but not trivial, generalization of "single" cosets. It can be considered as an action of a direct product $V \times U$ on $G$ with $(v, u) g=v g u^{-1}$. This action leads to non-trivial stabilizers

$$
(V \times U)_{g}=V \cap g U g^{-1}
$$

so, in a general case, double cosets contain fewer elements than $|V||U|$ and they can have different cardinalities, since, according to Eq. (1),

$$
|V g U|=\frac{|V||U|}{\left|V \cap g V g^{-1}\right|} .
$$

For the given subgroups $V, U$ the number of double cosets is given as [18]

$$
|V \backslash G / U|=\frac{|G|}{|V||U|} \sum_{g \in \mathcal{C}} \frac{|C(g) \cap V||C(g) \cap U|}{|C(g)|}
$$

where $C(g)$ denotes a conjugacy class of a element $g$ and $\mathcal{C}$ is a set of conjugacy classes representatives.

Among different applications of double cosets (see, for example, Altmann's monograph [19]) we are interested in action of $V \subseteq G$ on an orbit $G(x)$ in type $\widetilde{U}$. This orbit consists of elements labelled by representatives of left cosets in the following decomposition

$$
G=\underset{\bigcup^{j=1}}{|G / U|} g_{j} U
$$

The action of $V$ on $G(x)$ is similar to the action of $V$ on $G / U$, so orbits are labelled by double coset representatives [18]. In the trivial case $U=E$ it corresponds to the left regular action of $V$ on $G$, so to the right cosets.

The second important point is when we consider an action of $G$ on the Cartesian product $G(y) \times G(x)$ of two orbits with the stabilizers $G_{x}=U$ and $G(y)=V$. This action is defined as follows

$$
(g,(y, x)) \mapsto(g y, g x)
$$

and has the following properties ( $x$ and $y$ are fixed) [18]: 
- Each orbit contains an element of the form $(y, g x)$;

- The stabilizer of $(y, g x)$, given by Eq. (12), is $G_{y} \cap g G_{x} g^{-1}$;

- The mapping $\left(G(y, g x) \mapsto G_{y} g G_{x}\right.$ is a bijection, so orbits of the introduced action can be labelled by double coset representatives.

These properties yield relations between matrix elements of an operator $\mathcal{H}$ commuting with all symmetry operators $P(g)$. Since $P$ is a unitary representation then $P\left(g^{-1}\right)=P(g)^{\dagger}$ and for any basis vectors one obtains

$$
\langle x|\mathcal{H}| y\rangle=\left\langle x\left|\mathcal{H} P\left(g^{-1}\right) P(g)\right| y\right\rangle=\langle P(g) x|\mathcal{H}| P(g) y\rangle .
$$

Hence, if $g \in G_{y}$ then

$$
\langle x|\mathcal{H}| y\rangle=\langle P(g) x|\mathcal{H}| y\rangle .
$$

Therefore, all states $x^{\prime}$ in the orbit $G_{y}(x)=\left\{g x \mid g \in G_{y}\right\}$ result in the same value of the matrix element $\left\langle x^{\prime}|\mathcal{H}| y\right\rangle$. Recall that these orbits are labelled by representatives of double cosets $G_{y} \backslash G / G_{x}$. Viewing this problem as an action of $g \in G$ on the Cartesian product $G(y) \times G(x)$ we see that each orbit contains an element $(y, g x)$ so all matrix elements can be derived from the action of the Hamiltonian $\mathcal{H}$ on the orbit representative $|y\rangle$.

\section{Irreducible basis and matrix elements}

In many cases it is easy to decompose the space of states into subspaces labelled by good quantum numbers, i.e. by eigenvalues of operators commuting with a Hamiltonian. In the case of Heisenberg magnets with at most uniaxial anisotropy the total magnetization (eigenvalue of the projection of total spin) plays this role $[3,8]$. Moreover, the symmetry operators do not mix these subspaces, so in the further considerations we restrict ourselves to one of such subspaces $L$ with a base $\mathcal{B}$ regardless of the actual values of good quantum numbers. As the symmetry group $G$ we take only those symmetries of a Hamiltonian, which permute basis vectors $|x\rangle \in \mathcal{B}$, so a permutation representation $P: G \rightarrow \Sigma_{\mathcal{B}}$ (or, in other words, an action of $G$ on $\mathcal{B}$ ) is considered.

The action ${ }_{G} \mathcal{B}$ in question decomposes $\mathcal{B}$ into disjoint orbits

$$
G(x)=\{|y\rangle \in \mathcal{B}|| y\rangle=P(g)|x\rangle, g \in G\} .
$$

Taking into account the orbit type (the conjugacy class $\widetilde{U}$ containing $G_{x}$ ) all states $|x\rangle \in \mathcal{B}$ can be labelled by three indices: (i) a subgroup $U \subseteq G$ denoting the type of an orbit; (ii) a state $|x\rangle$ with a stabilizer $U$ representing an orbit $^{\dagger}$; (iii) an element $g \in G$ representing a coset $g U$ - due to the similarity of actions $G G(x)$ and $G(G / U)$ states in the orbit $G(x)$ are in one-to-one correspondence with the left cosets $G / U$. Within this frame states of $\mathcal{B}$ will be denoted as

IIf there are $N(U)$ orbits in a type $U$ then the orbits can be also identified by an index $l=1,2, \ldots, N(U)$. 


$$
|U x g\rangle=P(g)\left|U x e_{G}\right\rangle=P(g)|x\rangle \in G(x) \subseteq \mathcal{B}, \quad G_{x}=U .
$$

The representation $P$ restricted to an orbit $G(x)$ in type $U$ is a transitive representation $R^{G: U}$ and decomposes into irreps $\Gamma$ of $G$ according to Eq. (3)

$$
R^{G: U}=\bigoplus_{\Gamma} n\left(\Gamma, R^{G: U}\right) \Gamma, \quad 0 \leq n\left(\Gamma, R^{G: U}\right) \leq[\Gamma]
$$

The irreducible basis $\{|U x ; \Gamma r \gamma\rangle\}$ can be constructed separately for each orbit using Eq. (6) and its vector can be written as

$$
|U x ; \Gamma r \gamma\rangle=\sum_{j=1}^{|G / U|} b_{j}^{\Gamma r \gamma}(U)\left|U x g_{j}\right\rangle
$$

the elements $g_{j}, 1 \leq j \leq|G / U|$ with $g_{1}=e_{G}$, represents the left cosets $g U \in G / U$. The coefficients $b_{j}^{\Gamma r \gamma}$, determining a unitary transformation, can be expressed by matrix elements of the irrep $\Gamma$ and the reduction coefficients $A_{\gamma}^{U \Gamma r}$, see Eq. (8), and are expressed as (cf. $[10,13])$

$$
b_{j}^{\Gamma r \gamma}(U)=\left(\frac{[\Gamma]|U|}{|G|}\right)^{1 / 2} \sum_{\beta} \Gamma_{\gamma \beta}^{*}\left(g_{j}\right)\left(A_{\beta}^{U \Gamma r}\right)^{*} .
$$

Matrix elements of any operator, e.g. a Hamiltonian $\mathcal{H}$, commuting with all $P(g)$ are grouped in blocks labelled by $\Gamma$ and depend only on $U, x$, and $r^{\ddagger}$. They are given by the following formula [9]:

$$
h_{U x r, V y s}(\Gamma)=\left(\frac{|U|}{|V|}\right)^{1 / 2} \sum_{j=1}^{|G / U|}\left\langle U x g_{j}|\mathcal{H}| V y e_{G}\right\rangle B_{r s}^{U V \Gamma}\left(g_{j}\right),
$$

where

$$
B_{r s}^{U V \Gamma}\left(g_{j}\right)=\sum_{\alpha, \beta}\left(A_{\alpha}^{V \Gamma s}\right)^{*} \Gamma_{\alpha \beta}\left(g_{j}\right) A_{\beta}^{U \Gamma r} .
$$

Note that the irreducible basis is not used in this formula in the explicit way, so, in fact, one does not need to determine vectors $|U x ; \Gamma r \gamma\rangle$. Introducing the notion of double cosets $\operatorname{Vg} U[18,19]$ and taking into account Eq. (14) this formula can be modified as:

$$
h_{U x r, V y s}(\Gamma)=\left(\frac{|U|}{|V|}\right)^{1 / 2} \sum_{d=1}^{|V \backslash G / U|}\left\langle U x g_{d}|\mathcal{H}| V y e_{G}\right\rangle D_{r s}^{U V \Gamma}\left(g_{d}\right),
$$

where elements $g_{d}$ represent double cosets $V g_{d} U$, or orbits of the natural action ${ }_{V}(G / U)$, and $D_{r s}^{U V \Gamma}\left(g_{d}\right)=\sum_{g_{j} \in V g_{d} U} B_{r s}^{U V \Gamma}\left(g_{j}\right)$. However, as follows from the property (10), the coefficients $B_{r s}^{U V \Gamma}\left(g_{j}\right)$ are constant on the double coset $V g_{d} U$.

$\ddagger$ A Hamiltonian (and any other linear operator commuting with all $P(g)$ ) preserves the linear structure, i.e. labels $\Gamma$ and $\gamma$, but vectors $\left|\Gamma_{\gamma}\right\rangle$ from different orbits have to be combined to obtain eigenvectors of $\mathcal{H}$. 
Let $g_{j} \in V g_{d} U$. Therefore, there exist $v \in V$ and $u \in U$ such that $g_{j}=v^{-1} g_{d} u$ and

$$
\begin{gathered}
B_{r s}^{U V} \Gamma\left(g_{j}\right)=\sum_{\alpha, \alpha^{\prime}, \beta, \beta^{\prime}}\left(A_{\alpha}^{V \Gamma s}\right)^{*} \Gamma_{\alpha \alpha^{\prime}}\left(v^{-1}\right) \Gamma_{\alpha^{\prime} \beta^{\prime}}\left(g_{d}\right) \Gamma_{\beta^{\prime} \beta}(u) A_{\beta}^{U \Gamma r}= \\
\sum_{\alpha^{\prime}, \beta^{\prime}}\left(A_{\alpha^{\prime}}^{V \Gamma s}\right)^{*} \Gamma_{\alpha^{\prime} \beta^{\prime}}\left(g_{j}\right) A_{\beta^{\prime}}^{U \Gamma r} .
\end{gathered}
$$

It yields the following form of Eq. (19)

$$
h_{U x r, V y s}(\Gamma)=\left(\frac{|U|}{|V|}\right)^{1 / 2} \sum_{d=1}^{|V \backslash G / U|}\left|V\left(g_{d} U\right)\right|\left\langle U x g_{d}|\mathcal{H}| V y e_{G}\right\rangle B_{r s}^{U V \Gamma}\left(g_{d}\right)
$$

where $V\left(g_{d} U\right)$ is an orbit obtained by the action of $V$ on the set of left cosets $G / U$. Each left coset has the cardinality $|U|$, then $\left|V\left(g_{d} U\right)\right|=\left|V g_{d} U\right| /|U|$ and, finally,

$$
h_{U x r, V y s}(\Gamma)=(|U||V|)^{-1 / 2} \sum_{d=1}^{|V \backslash G / U|}\left|V g_{d} U\right|\left\langle U x g_{d}|\mathcal{H}| V y e_{G}\right\rangle B_{r s}^{U V \Gamma}\left(g_{d}\right) .
$$

These equations simplify for the unit representation $\Gamma_{0}$. The matrix elements $\Gamma_{0}(g)$, the reduction coefficients $A^{U \Gamma}$ and their products $B^{U V \Gamma}$ are equal to 1 , so

$$
\left|U x ; \Gamma_{0}\right\rangle=|G / U|^{-1 / 2} \sum_{j=1}^{|G / U|}\left|U x g_{j}\right\rangle
$$

The operator matrix elements are

$$
h_{U x, V y}\left(\Gamma_{0}\right)=\left(\frac{|U|}{|V|}\right)^{1 / 2} \sum_{j=1}^{|G / U|}\left\langle U x g_{j}|\mathcal{H}| V y e_{G}\right\rangle .
$$

If there is only one double coset, represented by $e_{G}$, then all left coset representatives belong to it and

$$
h_{U x, V y}\left(\Gamma_{0}\right)=\left(\frac{|G|}{|U||V|}\right)^{1 / 2}\left\langle U x e_{G}|\mathcal{H}| V y e_{G}\right\rangle
$$

so it is enough to calculate matrix elements for orbit representatives.

Both equations, (17) and (20), lead to two "technical" problems: (i) calculation of the coefficients $B\left(g_{j}\right)$ and (ii) determination of $g_{d}$ for given $V, y, U, x$, and $\mathcal{H}$ and then calculation of non-zero elements $\left\langle U x g_{d}|\mathcal{H}| V y e_{G}\right\rangle$. The first problem is discussed in the next paper [17] for the dihedral groups $D_{N}$, whereas solution of the second one should be made within the frame of algebraic combinatorics (generation of orbit representatives $|y\rangle$ with a given stabilizer $V$, an action of $V$ on $G / U$, etc.) and computer methods (storing of configurations, action of a Hamiltonian, searching configurations $\mathcal{H}\left|V y e_{G}\right\rangle$ or, equivalently, $\left.\left\langle U x g_{d}\right| \mathcal{H}\right)$. Possible solutions of the latter problem are presented elsewhere $[15,16]$. 


\section{Partitions}

Before we apply formulas presented above to spin rings with the symmetry $D_{N}$, we analyze the space of states using the total $z$-projection operator $S^{z}$

$$
S^{z}\left|m_{1} m_{2} \ldots m_{N}\right\rangle=\left(\sum_{j=1}^{N} m_{j}\right)\left|m_{1} m_{2} \ldots m_{N}\right\rangle .
$$

It is assumed that this operator commutes with a Hamiltonian, so its eigenvalue, the magnetization $-N s \leq M \leq N s$, is a good quantum number. Therefore, subbases $\mathcal{B}_{M}=\left\{\mu: S^{z} \mu=\bar{M} \mu\right\}$ span eigenspaces $L_{M}$ of the Hamiltonian, and all considerations can be limited to these subspaces. Moreover, due to the time-reversal symmetry, it suffices to investigate the cases $M \geq 0$.

The operator $S^{z}$ commutes with the whole symmetric group $\Sigma_{N}$ and the value of $M$ depends on a non-ordered partition $[k]=\left[k_{-s}, k_{-s+1}, \ldots, k_{s}\right]$ of $N$ into $2 s+1$ non-negative parts. Each $k_{l}, l \in[-s, s]=\{-s,-s+1, \ldots, s\}$, denotes a number of projections equal to $l$, so the magnetization equals

$$
M=\sum_{l \in[-s, s]} l k_{l} .
$$

A number of configurations for a given partition $[k]$ follows from the relation between an orbit order and its stabilizer, see Eq. (1). In the case considered, a stabilizer of each configuration $\mu$ with the partition $[k]$ is isomorphic to the Young subgroup

$$
\Sigma_{[k]}=\bigotimes_{l \in[-s, s]} \Sigma_{k_{l}}
$$

Since configurations with the same partition $[k]$ form an orbit $\mathcal{B}_{[k]}$ of the symmetric group $\Sigma_{N}$, then

$$
\left|\mathcal{B}_{[k]}\right|=\frac{\left|\Sigma_{N}\right|}{\left|\Sigma_{[k]}\right|}=\frac{N !}{\prod_{l \in[-s, s]} k_{l} !},
$$

so it is given by the polynomial coefficient [8]. The relations between $M \geq 0$ and non-ordered partitions $[k]$ for six spins $s=1$ are collected in Table $\mathbf{I}$. Two important remarks are in place. At first, we note that the action of bilinear terms $s_{j}^{+} s_{j^{\prime}}^{-}$can modify at most four parts of the partition. Namely, if in the original configuration $m_{j}=a$ and $m_{j^{\prime}}=b$ and $b \geq a+2$ or $b<a$, the final configuration (obtained by the action of $s_{j}^{+} s_{j^{\prime}}^{-}$) contains $m_{j}=a+1$ and $m_{j^{\prime}}=b-1$. Therefore, the partition $\left[\ldots k_{a} k_{a+1} \ldots k_{b-1} k_{b} \ldots\right]$ is modified to $\left[\ldots k_{a}-1 k_{a+1}+1 \ldots k_{b-1}+\right.$ $\left.1 k_{b}-1 \ldots\right]$. In the special cases, especially for small spin numbers, $|a-b|=0$ or 1 which yields the modification of only three or even none of parts $k_{i}, i=$ $0,1, \ldots, 2 s$. For example, $s_{2}^{+} s_{4}^{-}|+0-0\rangle=|++--\rangle$, so the partition [121] is modified to [202]. On the other hand, the action of $s_{2}^{+} s_{1}^{-}$does not modify the partition. Therefore, one can construct a graph (with loops) with vertices labelled 
TABLE I

Relations between $M \geq 0$ and non-ordered partitions for $N=6$ and $s=1$

\begin{tabular}{c|c|c|c}
\hline \hline$M$ & {$[k]$} & $\left|\mathcal{B}_{[k]}\right|$ & Representative of $\mathcal{B}_{[k]}$ \\
\hline 6 & {$[006]$} & 1 & $|++++++\rangle$ \\
\hline 5 & {$[015]$} & 6 & $|0+++++\rangle$ \\
\hline 4 & {$[105]$} & 6 & $|-+++++\rangle$ \\
& {$[024]$} & 15 & $|00++++\rangle$ \\
\hline 3 & {$[114]$} & 30 & $|-0++++\rangle$ \\
& {$[033]$} & 20 & $|--++++\rangle$ \\
\hline 2 & {$[204]$} & 15 & $|-00+++\rangle$ \\
& {$[123]$} & 60 & $|-000++\rangle$ \\
& {$[042]$} & 15 & $|-000+++\rangle$ \\
\hline 1 & {$[213]$} & 60 & $|---+++\rangle$ \\
& {$[132]$} & 60 & $|--00++\rangle$ \\
& {$[051]$} & 6 & $|-0000+\rangle$ \\
\hline 0 & {$[303]$} & 20 &
\end{tabular}

by non-ordered partitions $[k]$ and edges joining such pairs of vertices, $[k]$ and $\left[k^{\prime}\right]$ say, that there is a term $s_{j}^{+} s_{j^{\prime}}^{-}$in the operator considered, which transforms a configuration in $\mathcal{B}_{[k]}$ into a configuration in $\mathcal{B}_{\left[k^{\prime}\right]}$. The edges can be treated as non-directed, since Hermitian operators contain both terms $s_{j}^{+} s_{j^{\prime}}^{-}$and $s_{j}^{-} s_{j^{\prime}}^{+}$. In the simplest case $s=1 / 2$ only loops are admissible, since the magnetization $M$ uniquely determines a non-ordered partition $[k]=[N / 2-M, N / 2+M]$. In the example considered here $(s=1)$ a graph is linear and its vertices can be labelled by $k_{0}$. In a general case the graph obtained has a more complicated structure. For example, the case of six spins $s=3 / 2$ and $M=4$ yields the following structure (the loops are omitted, since they are always admissible in this case) ${ }^{\hat{g}}$ :

$$
[0,0,5,1]-[0,1,3,2]<{ }_{[0,2,1,3]}^{[1,0,2,3]}
$$

The second important point follows from the notion of strata (types of orbits) introduced in Sec. 2.1. In the case considered a stratum is labelled by an ordered partition $[\kappa]=\left[\kappa_{-s}, \ldots, \kappa_{s}\right]$ with the additional condition $\kappa_{l} \geq \kappa_{l+1}$ for all $-s \leq l<s$. The trailing zeroes (the last parts equal zero) are, as usual, omitted

\footnotetext{
${ }^{\S}$ For a given partition $[k]$ a loop is admissible if there is at least one pair of non-zero adjoining parts $k_{l}, k_{l+1}$.
} 
and a number of non-zero parts is called a partition length. In the case considered there are seven ordered partitions of $N=6$ with no more than $2 s+1=3$ parts: $[6],[5,1],[4,2],[4,1,1],[3,3],[3,2,1]$, and $[2,2,2]$. All orbits $\mathcal{B}_{[k]}$ in type $[\kappa]$ decompose into orbits of $P(G) \equiv \bar{G} \subset \Sigma_{N}$ in the same way, because they are related to double cosets $\bar{G} \backslash \Sigma_{N} / \Sigma_{[\kappa]}$ [18]. Therefore, it is enough to consider ordered partitions and then apply the results to non-ordered ones. For example, the ordered partition $[N]$ represents $2 s+1$ non-ordered partitions and all of them correspond to fully symmetric states $|m m \ldots, m\rangle, m \in[-s, s]$. A more detailed presentation of partition-related questions can be found in some earlier papers [8], works on numerical algorithms $[15,16]$, and, the last but not least, Kerber's monograph [18].

\section{Example}

A system of four spins $s=1$ with the symmetry group $G=D_{4}$ is very simple, but some characteristics features may be observed even in this case. In the discussion presented below some results of the previous works [8] and the following paper [17] are exploited. The Schönflies symbols of groups and their elements are used [20].

There are eight classes of conjugated subgroups (see, for example, [17] or [18]), but only five of them can be stabilizers of magnetic configurations (see Sec. 2.3). Besides trivial stabilizers $D_{4}=\left\{E, C_{4}, C_{2}, C_{4}^{-1}, U_{0}, U_{1}, U_{2}, U_{3}\right\}$ and $C_{1}=\{E\}$ they are

$$
D_{2}^{0}=\left\{E, C_{2}, U_{0}, U_{2}\right\}, \quad D_{1}^{0}=\left\{E, U_{0}\right\}, \quad D_{1}^{1}=\left\{E, U_{1}\right\}
$$

If we consider a Heisenberg-like Hamiltonian with at most uniaxial anisotropy, then the magnetization is a good quantum number and subspaces with $M=$ $0, \pm 1, \pm 2, \pm 3, \pm 4$ can be considered separately. The case $M=4(M=-4)$ is the simplest one, since there is the unique Ising configuration $|++++\rangle(|----\rangle)$. On the other hand, there are 19 configurations with $M=0$ which can be decomposed into five orbits. Their stabilizers (all are different) and representatives are as follows:

$$
\begin{gathered}
D_{4}:|1\rangle=|0000\rangle, \quad D_{2}^{0}:|2\rangle=|+-+-\rangle, \quad D_{1}^{0}:|3\rangle=|+0-0\rangle, \\
D_{1}^{1}:|4\rangle=|++--\rangle, \quad C_{1}:|5\rangle=|+00-\rangle .
\end{gathered}
$$

Orders of these orbits are: $1,2,4,4$, and 8 . Note that orbits in types $D_{2}^{0}$ and $D_{1}^{1}$ are labelled by non-ordered partition [202], whereas the orbits in type $D_{1}^{0}$ and $C_{1}$ by [1 21$]$. Since there is only one orbit in each type, then they need not be distinguished by the partition label and, therefore, it will be omitted in further considerations. In the next step we decompose the transitive representations into irreps:

$$
R^{D_{4}: D_{4}}=A_{1}
$$




$$
\begin{aligned}
& R^{D_{4}: D_{2}^{0}}=A_{1} \oplus B_{1}, \\
& R^{D_{4}: D_{1}^{0}}=A_{1} \oplus B_{1} \oplus E, \\
& R^{D_{4}: D_{1}^{1}}=A_{1} \oplus B_{2} \oplus E \\
& R^{D_{4}: C_{1}}=A_{1} \oplus A_{2} \oplus B_{1} \oplus B_{2} \oplus 2 E .
\end{aligned}
$$

Let us consider the isotropic antiferromagnetic Heisenberg Hamiltonian with the nearest and next-nearest interactions

$$
\mathcal{H}=J_{1}\left(s_{1} s_{2}+s_{2} s_{3}+s_{3} s_{4}+s_{4} s_{1}\right)+2 J_{2}\left(s_{1} s_{3}+s_{2} s_{4}\right)
$$

where $J_{1}>0$ and the factor 2 is introduced to mimic four pairs of next-nearest-neighbors. Note that in the special case $J_{1}=2 J_{2}=2$ we have $\mathcal{H}=\boldsymbol{S}^{2}-8$. The eigenvalues of $\boldsymbol{S}^{2}$ are known and they can be easily related to irreps [8, 15], as shown in Table II. In a general case, the decompositions of transitive representations lead to the following classifications of states:

- Five states are totally symmetric $\left(\Gamma=A_{1}\right)$; they are linear combinations of all states with $M=0$; they are related to even total spin numbers;

- Only one state has symmetry $A_{2}$ obtained from the orbit with a stabilizer $C_{1}$ and related to $S=1$;

- Three states are labelled by $B_{1}$ (orbits 2, 3, and 5); they should give $S=$ $1,2,3$;

- The irrep $B_{2}$ labels two states (orbits 4 and $5, S=0,2$ );

- The two-dimensional irrep $E$ labels doubly degenerated levels with states $|E x\rangle$ and $|E y\rangle$. It appears in orbits 3, 4, and 5 (twice) and correspond to $S=3,2$, and 1 (two states).

\section{TABLE II}

Eigenvalues of $\boldsymbol{S}^{2}$, corresponding energies $\mathcal{E}$ for $J_{1}=$ $2 J_{2}=2$, and symmetries of energy levels (i.e. irreps labelling them).

\begin{tabular}{c|c|c|c}
\hline \hline$S$ & $S(S+1)$ & $\mathcal{E}=S(S+1)-8$ & $\Gamma$ \\
\hline 4 & 20 & 12 & $A_{1}$ \\
3 & 12 & 4 & $B_{1} \oplus E$ \\
2 & 6 & -2 & $2 A_{1} \oplus B_{1} \oplus B_{2} \oplus E$ \\
1 & 2 & -6 & $A_{2} \oplus B_{1} \oplus 2 E$ \\
0 & 0 & -8 & $2 A_{1} \oplus B_{2}$
\end{tabular}

Let us consider the states labelled by $B_{2}$. Non-zero matrix elements are possible only between the fourth and fifth orbit, so they will be denoted $h_{x y}$ with $x, y=4,5$. For all one-dimensional irreps we have $A^{U \Gamma}=1$ if $\Gamma$ appears in the 
decomposition of $R^{G: U}$ (indices $\gamma$ and $r$ may be omitted since both of them have only one value). Therefore, see Eq. (18), $B^{U V \Gamma}\left(g_{j}\right)=\Gamma\left(g_{j}\right)$. In the case considered we have

$$
\begin{aligned}
& B_{2}(E)=B_{2}\left(C_{2}\right)=B_{2}\left(U_{1}\right)=B_{2}\left(U_{3}\right)=+1 \\
& B_{2}\left(C_{4}\right)=B_{2}\left(C_{4}^{-1}\right)=B_{2}\left(U_{0}\right)=B_{2}\left(U_{2}\right)=-1 .
\end{aligned}
$$

Next we have to consider double cosets $V \backslash D_{4} / U$ for $U, V=D_{1}^{1}, C_{1}$ ฯ. For $U=D_{1}^{1}$ there are four left cosets represented by $E, C_{4}, C_{2}$, and $C_{4}^{-1}$. They are also double cosets for $V=C_{1}$, but they form three double cosets for $V=D_{1}^{1}$ : the action of $U_{1}$ merges the second and the fourth coset and, in this case, one obtains

$$
\left|D_{1}^{1} E D_{1}^{1}\right|=\left|D_{1}^{1} C_{2} D_{1}^{1}\right|=2, \quad\left|D_{1}^{1} C_{4} D_{1}^{1}\right|=4 .
$$

These formulas are also valid for $U=C_{1}$ and $V=D_{1}^{1}$. For $U=V=C_{1}$ there are, of course, eight double cosets represented by all elements of the group $D_{4}$.

In the case $U=V=D_{1}^{1}$ one has to consider three elements $\langle x|\mathcal{H}| y\rangle$ with $y=|++--\rangle$ and $x=y, x=P\left(C_{4}\right) y$, and $x=P\left(C_{2}\right) y$, i.e.

$$
\langle++--|\mathcal{H}|++--\rangle, \quad\langle+--+|\mathcal{H}|++--\rangle, \quad\langle--++|\mathcal{H}|++--\rangle .
$$

It can be noticed that the last two are equal to zero, since the Hamiltonian considered does not contain the operator $s_{2}^{-} s_{2}^{-}$(necessary to change $m_{2}=+1$ to $m_{2}^{\prime}=-1$ ). The first element is non-zero only for the diagonal terms $s_{j}^{z} s_{k}^{z}$ and gives the value $2 J_{2}\left(m_{1} m_{3}+m_{2} m_{4}\right)=-4 J_{2}$. Since $B_{2}\left(C_{2}\right)=1$, this is the value of the matrix element $h_{44}$.

Let us consider now $V=C_{1}$. There are four terms which should be considered (for $g_{d}=C_{4}^{j}, j=0,1,2,3$ ):

$$
\begin{array}{ll}
\langle++--|\mathcal{H}|+00-\rangle, & \langle+--+|\mathcal{H}|+00-\rangle \\
\langle--++|\mathcal{H}|+00-\rangle, & \langle-++-|\mathcal{H}|+00-\rangle .
\end{array}
$$

Only the first one is non-zero, since the others needs the term $s_{4}^{+} s_{4}^{+}$or $s_{1}^{-} s_{1}^{-}$to be present in the Hamiltonian. This non-zero term corresponds to $\left(s_{2}^{+} s_{3}^{-}\right) / 2$ and, therefore, give the value $J_{1}$. It has to be multiplied by $\left|C_{1} E D_{1}^{1}\right|=2$ and divided by $(|U| /|V|)^{1 / 2}=\sqrt{2}$.

To finish the calculations it is enough to consider the case $U=V=C_{1}$, but we start with $U=C_{1}$ and $V=D_{1}^{1}$ to check the value just obtained. The double cosets are again represented by $g_{d}=C_{4}^{j}(j=0,1,2,3)$, so four terms have to be considered:

$$
\begin{array}{cc}
\langle+00-|\mathcal{H}|++--\rangle, & \langle 00-+|\mathcal{H}|++--\rangle, \\
\langle 0-+0|\mathcal{H}|++--\rangle, & \langle-+00|\mathcal{H}|++--\rangle .
\end{array}
$$

IIf classes $\widetilde{U}$ are labelled by an index $j$ then it is enough to consider cosets $U_{j} \backslash G / U_{j^{\prime}}$ for $j^{\prime} \leq j$ since the Hamiltonian matrix has to be Hermitian. 
Only the first one is non-zero and equals $J_{1}$ and has to be multiplied by $\sqrt{2}$, as in the previous case. Therefore, the Hamiltonian matrix is symmetric.

In the last case $U=V=C_{1}$ one has to consider all eight elements since every double coset $V g_{d} U=\left\{g_{d}\right\}$. Note that in this case $|U|=|V|=\left|V g_{d} U\right|=1$. It can be noticed that only three scalar products are non-zero:

$$
\langle+00-|\mathcal{H}|+00-\rangle, \quad\langle+-00|\mathcal{H}|+00-\rangle, \quad\langle 00+-|\mathcal{H}|+00-\rangle .
$$

The first is obtained for $g_{d}=E$ and has the value $-J_{1}$, whereas the others equal $2 J_{2}$ and are related to $g_{d}=U_{0}, U_{2}$, so they have to be multiplied by $B_{2}\left(g_{d}\right)=-1$ in this case. As the results one obtains a $2 \times 2$ symmetric matrix

$$
\left(\begin{array}{cr}
-4 J_{2} & \sqrt{2} J_{1} \\
\sqrt{2} J_{1} & -J_{1}-4 J_{2}
\end{array}\right)
$$

with eigenvalues

$$
\mathcal{E}_{1}=-2 J_{1}-4 J_{2}, \quad \mathcal{E}_{2}=J_{1}-4 J_{2} .
$$

For $J_{1}=2 J_{2}=2$ one has $\mathcal{E}_{1}=-8$ and $\mathcal{E}_{2}=-2$, which agrees with Table II. The eigenvectors are

$$
\begin{aligned}
& \left|B_{2} \mathcal{E}_{1}\right\rangle=\left(\left|D_{1}^{1} 4 ; B_{2}\right\rangle-\sqrt{2}\left|C_{1} 5 ; B_{2}\right\rangle\right) / \sqrt{3} \\
& \left|B_{2} \mathcal{E}_{2}\right\rangle=\left(\sqrt{2}\left|D_{1}^{1} 4 ; B_{2}\right\rangle+\left|C_{1} 5 ; B_{2}\right\rangle\right) / \sqrt{3}
\end{aligned}
$$

where vectors $\left|U x ; B_{2}\right\rangle$ for $U=D_{1}^{1}, C_{1}$ and $x=4,5$ ( $x$ labels orbits) can be determined according to Eqs. (15) and (16).

\section{Final remarks and conclusions}

The formula (20) and its special case (22) make a basis for construction of operator matrices, especially for a Hamiltonian $\mathcal{H}$. It has to be stressed that one investigates only the action of $\mathcal{H}$ on orbit representatives $\left|V y e_{G}\right\rangle$ or, strictly speaking, elements $\left\langle U x g_{d}|\mathcal{H}| V y e_{G}\right\rangle$ for all representatives $g_{d}$ of double cosets $V \backslash G / U$. At the first glance both tasks, determination of $\mathcal{H}|y\rangle$ and calculation of $\langle x|\mathcal{H}| y\rangle$, are equivalent. And it is true from the point of view of the mathematical theory and the physical meaning of the formulas. However, they are different tasks when one considers their actual realization, e.g. implementation of algorithms.

Let us assume that a given Hamiltonian can be written as a sum of such terms $\mathcal{H}_{k}$ that $\mathcal{H}_{k}|y\rangle$ is exactly one configuration. In the case of spin systems it means that one of these terms, say $\mathcal{H}_{0}$, contains only $s_{j}^{z}$ operators, since configurations are their eigenvectors. For example, the Heisenberg interaction of the nearest neighbors in $N$-element ring leads to

$$
\mathcal{H}_{0}=J \sum_{j=1}^{N} s_{j}^{z} s_{j+1}^{z}, \quad N+1 \equiv 1 .
$$


Each of all $2 N$ other terms $s_{j}^{+} s_{j+1}^{-}$and $s_{j}^{-} s_{j+1}^{+}$produces a new (and different) configuration or an empty state |\rangle . Therefore, for each $\left|y_{k}\right\rangle=\mathcal{H}_{k}|y\rangle, k=$ $0,1,2, \ldots, 2 N$, one has to perform the following steps: (i) determination of its stabilizer $U$; (ii) search for an orbit (in fact, its representative $x$ ), to which $\left|y_{k}\right\rangle$ belongs; (iii) determination of a double-coset representative $g_{d}$ such that $P\left(g_{d}\right)|x\rangle=\left|y_{k}\right\rangle$; (iv) addition of the product $\left|V g_{d} U\right|\left\langle U x g_{d}|\mathcal{H}| V y e_{G}\right\rangle B_{r s}^{U V \Gamma}\left(g_{d}\right)$ to $h_{U x r, V y s}(\Gamma)$. In this way the matrix $\mathcal{H}$ is constructed column by column.

In the second approach one can construct this matrix by elements. For a given irrep $\Gamma$ these elements are labelled by $U, V, x, y, r$, and $s$. Therefore, these indices are fixed in further calculations, which are performed as follows: (i) take all representatives $g_{d}$ of double cosets $V g_{d} U \in V \backslash G / U$ (they are known from the group-theoretical considerations); (ii) construct the state $P\left(g_{d}\right)|x\rangle$; (iii) check if the element $\left\langle U x g_{d}|\mathcal{H}| V y e_{G}\right\rangle$ is non-zero and, if yes, calculate it; (iv) add the product $\left|V g_{d} U\right|\left\langle U x g_{d}|\mathcal{H}| V y e_{G}\right\rangle B_{r s}^{U V \Gamma}\left(g_{d}\right)$ to $h_{U x r, V y s}(\Gamma)$.

This approach has some advantages. At first, one determines only the upper (or lower) part and the diagonal of the matrix for Hermitian (symmetric) operators. Moreover, in some cases it can be easily checked whether the configuration $P\left(g_{d}\right)|x\rangle$ can be obtained from the configuration $|y\rangle$ acting with a given Hamiltonian. For example, configurations of systems with $s=1 / 2$ can be stored as binary numbers $b_{n-1} b_{n-2} \ldots b_{2} b_{1} b_{0}$, where $n$ is the length of a machine word $(n=32$ or 64 , as usual; one can use an array of words if $N>n$ ) and $b_{j}=m_{j}+1 / 2$ for $m_{j}= \pm 1 / 2$. For two given configurations, say $b_{n-1} \ldots b_{1} b_{0}$ and $b_{n-1}^{\prime} \ldots b_{1}^{\prime} b_{0}^{\prime}$, one calculates the bit-wise "exclusive or" (XOR)

$$
c_{j}=b_{j} \operatorname{XOR} b_{j}^{\prime}=\left\{\begin{array}{cc}
0 & \text { if } b_{j}=b_{j}^{\prime} \\
1 & \text { otherwise }
\end{array}\right.
$$

If the obtained number $c_{n-1} \ldots c_{1} c_{0}$ has only two non-zero digits $c_{k}$ and $c_{k+d}$, then $\left|g_{d} x\right\rangle=s_{k}^{+} s_{k+d}^{-}|y\rangle$ or $s_{k}^{-} s_{k+d}^{+}|y\rangle$. In both cases $\left\langle g_{d} x|\mathcal{H}| y\right\rangle=J / 2$ if the Hamiltonian $\mathcal{H}$ contains interactions $J s_{k} s_{k+d}$. In the case $s>1 / 2$, the calculations are not so simple, but, nevertheless, faster than direct determination of configurations $s_{k}^{+} s_{k+d}^{-}|y\rangle$, when one has to check if $m_{k}<s$ and $m_{k+d}>-s$. Such an approach is especially useful in the case of mesoscopic rings or linear chains with the periodic boundary conditions, whereas in the case of two- and three-dimensional systems the first solution may be more adequate. Let us consider a $d$-dimensional (hyper)cubic lattice with the Born-von Kármán period $N$ in each direction. Then its (finite) space group has $(2 N)^{d} d$ ! elements [22] and it is the length of most orbits, since they belong to the generic stratum. On the other hand, the Heisenberg Hamiltonian with the nearest neighbor interactions has $2 d N^{d}$ terms $s_{j}^{+} s_{j^{\prime}}^{-}$, which may produce new configurations $\left|y_{k}\right\rangle$. Therefore, a loop over double coset representatives is longer than a loop over all Hamiltonian terms, since $\left[(2 N)^{d} d !\right] /\left(2 d N^{d}\right)=2^{d-1}(d-1)$ ! However, an introduction of additional terms to the Hamiltonian or consideration of $\boldsymbol{S}^{2}$ operator (with $N^{d}\left(N^{d}-1\right)$ 
terms $\left.s_{j}^{+} s_{j^{\prime}}^{-}\right)$may change this comparison significantly.

The above considerations are elicited by possible "practical" applications of the formulas obtained. However, they also describe general properties of an operator $\mathcal{H}$ commuting with symmetry operators $P(g), g \in G$. Matrix elements $\langle x|\mathcal{H}| y\rangle$ of such operators are constant on pairs belonging to the same orbit determined by the action of $G$ on a Cartesian product of orbits $G(y) \times G(x)$; these orbits are labelled by double coset representatives $g_{d}$. When a discrete problem of the finite action ${ }_{G} \mathcal{B}$ is linearized, i.e. (vector) irreducible representations $\Gamma$ are introduced, then matrix elements in the symmetry adapted basis $\{|U x ; \Gamma r \gamma\rangle\}$ can be determined using the reduction coefficients $A_{\gamma}^{U \Gamma r}$. All model parameters depending only on the problem symmetry can be determined before calculations of matrix elements and applied to all models/Hamiltonians with the same symmetry. In the case of simple groups, like cyclic or dihedral ones, these parameters can be expressed in the analytic form for the whole family of groups. In the next paper [17] the results obtained for the dihedral groups $D_{N}$ are presented. These groups are important in the investigations of mesoscopic rings and chains with the periodic boundary conditions. It can be proven [22] that a (finite) space group of the $d$-dimensional (hyper)cubic lattice is a wreath product $D_{N}<\Sigma_{d}$ and, therefore, the results obtained for $D_{N}(d=1)$ are useful in the investigations of two- and three-dimensional lattices $(d=2,3)$.

\section{Acknowledgments}

This work is partially supported bythe State Committee for Scientific Research within the project No. 2 P03B 074 19. The author is indebted to Prof. A. Kerber for fruitful discussions. The valuable remarks of Prof. G. Kamieniarz and Dr. S. Wałcerz are also acknowledged.

\section{References}

[1] H.A. Bethe, Z. Phys. 71, 205 (1931); L. Hulthén, Arkiv Mat. Astron. Fys. A 26, 1 (1938).

[2] J.C. Bonner, M.E. Fisher, Phys. Rev. A 135, 640 (1964); J. Oitmaa, D.D. Betts, Can. J. Phys. 56, 897 (1978).

[3] W. Marshall, Proc. R. Soc. A 232, 48 (1955); E. Lieb, D. Mattis, J. Math. Phys. 3, 749 (1962); P.L. Iske, W.J. Caspers, Physica A 142, 360 (1987); E. Dagatto, A. Moreo, Phys. Rev. B 38, 5087 (1989); K. Fabricius, U. Löw, K.-H. Müter, P. Ueberholz, Phys. Rev. B 44, 7476 (1991), G. Kamieniarz, H.W.J. Blöte, J. Phys. A, Math. Gen. 26, 201 (1993); G. Kamieniarz, Phase Trans. 57, 105 (1996).

[4] M. Affronte, J.C. Lasjaunias, A. Cornia, A. Caneschi, Phys. Rev. B 60, 1161 (1999) 
[5] D. Gatteschi, A. Caneschi, L. Pardi, R. Sessoli, Science 265, 1054 (1994); A. Lascialfari, D. Gatteschi, A. Cornia, U. Balucani, M.G. Pini, A. Rettori, Phys. Rev. B 57, 1115 (1998); A. Caneschi, D. Gatteschi, C. Sangregorio, R. Sessoli, L. Sorace, A. Cornia, M.A. Novak, C. Paulsen, W. Wernsdorfer, J. Magn. Magn. Mater. 200, 182 (1999)

[6] J.E. Hirsh, R.L. Sugar, D.J. Scalapino, R. Blankenbecler, Phys. Rev. B 26, 5033 (1982); C. Kalle, V. Winkelmann, J. Stat. Phys. 28, 639 (1982); S. Wansleben, J.G. Zabolitzky, C. Kalle, J. Stat. Phys. 37, 271 (1984); H.W.J. Blöte, E. Luijten, J.R. Heringa, J. Phys. A, Math. Gen. 28, 6289 (1995).

[7] P. Pawlicki, J. Rogiers, Physica A 214, 277 (1995); P. Pawlicki, G. Kamieniarz, L. Dębski, Physica A 242, 290 (1997).

[8] W. Florek, Acta Magnetica II, 43 (1985); V, 145 (1988); VIII, 25 (1991).

[9] G. Kamieniarz, R. Matysiak, W. Florek, S. Wałcerz, J. Magn. Magn. Mater. 203, 271 (1999).

[10] W. Florek, Acta Phys. Pol. A 96, 699 (1999).

[11] L.D. Faddeev, L.A. Takhtajan, Zapiski Nauchnykh Seminarov LOMI 109, 134 (1981); R. Olchawa, Physica B 291, 29 (2000).

[12] B. Lulek, T. Lulek, Acta Phys. Pol. A 66, 149 (1984) and the references cited therein.

[13] B. Lulek, T. Lulek, J. Phys. A, Math. Gen. 17, 3077 (1984).

[14] B. Lulek, T. Lulek, R. Chatterjee, J. Biel, Can. J. Phys. 63, 1061 (1985); B. Lulek, T. Lulek, J. Biel, R. Chatterjee, Can. J. Phys. 63, 1065 (1985); B. Lulek, T. Lulek, Acta Phys. Pol. A 71, 869 (1987); W. Florek, T. Lulek, J. Phys. A, Math. Gen. 20, 1921 (1987); T. Lulek, Acta Phys. Pol. A 80, 769 (1991); S. Wałcerz, T. Lulek, Acta Phys. Pol. A 80, 781 (1991); S. Wałcerz, Acta Magnetica X, 13 (1994/95).

[15] W. Florek, Comp. Phys. Commun. 138, 264 (2001).

[16] S. Bucikiewicz, W. Florek, Comp. Meth. Sci. Tech. 7, 27 (2001); W. Florek, Comp. Meth. Sci. Tech. 7, 41 (2001).

[17] S. Bucikiewicz, L. Debski, W. Florek, Acta Phys. Pol. A, in press.

[18] A. Kerber, Algebraic Combinatorics via Finite Group Actions, BI Wissenshaftsverlag, Mannheim-Wien-Zürich 1991.

[19] S.L. Altmann, Induced Representations in Crystals and Molecules, Academic Press, London 1977.

[20] C.J. Bradley, A.P. Cracknell, The Mathematical Theory of Symmetry in Solids, Clarendon, Oxford 1972.

[21] T. Lulek, Acta Phys. Pol. A 57, 415 (1980); D.B. Litvin, J. Math. Phys. 23, 337 (1982). S.C. Chen, D.J. Newman, J. Phys. A, Math. Gen. 15, 3383 (1982); T. Lulek, M. Szopa, J. Phys. A, Math. Gen. 23, 677 (1990); S. Wałcerz, T. Lulek, Acta Phys. Pol. A 90, 781 (1991); A. Kerber, in: Symmetry and Structural Properties of Condensed Matter, Eds. W. Florek, T. Lulek, M. Mucha, World Sci., Singapore 1991, p. 3.

[22] W. Florek, T. Lulek, J. Phys. A, Math. Gen. 26, 2153 (1993). 